\title{
Pulmonary embolism in an adolescent girl with negative ACLA systemic lupus erythematosus (SLE): a case report
}

\author{
Seyed Hesamedin Nabavizadeh ${ }^{1}$, Nazanin Farahbakhsh², Ali Fazel $^{3}$, Hamidreza Houshmand ${ }^{3}$, Amir \\ Anushiravani $^{4}$
}

\begin{abstract}
${ }^{1}$ M.D., Professor, Department of Immunology and Allergy, Faculty of Medicine, Allergy Research Center, Shiraz University of Medical Sciences, Shiraz, Iran

${ }^{2}$ M.D., Faculty Member, Department of Pediatrics, Faculty of Medicine, Allergy Research Center, Shiraz University of Medical Sciences, Shiraz, Iran

${ }^{3}$ M.D., Faculty Member, Department of Immunology and Allergy, Faculty of Medicine, Allergy Research Center, Shiraz University of Medical Sciences, Shiraz, Iran

${ }^{4}$ M.D., Faculty Member, Department of Internal Medicine, Faculty of Medicine, Allergy Research Center, Shiraz University of Medical Sciences, Shiraz, Iran
\end{abstract}

Type of article: Case report

\begin{abstract}
Pulmonary involvement is a common manifestation in systemic lupus erythematosus (SLE), whereas pulmonary thromboembolism (PTE) is rarely seen in SLE. PTE related to anti-phospholipid antibody syndrome (APS) is also a rare disease. We have reported a 13-year-old female diagnosed with SLE Two years ago, who is being treated with hydroxychloroquine and prednisolone. She presented with shortness of breath, dry cough, and fever about two weeks prior to admission. She was initially admitted with the diagnosis of pneumonia, but no clinical improvement was seen she was given antibiotics. Hemoptysis was added to her symptoms, so spiral high resolution computed tomography (HRCT) of the lungs was requested, and it indicated patchy consolidations bilaterally. With suspicion of pulmonary thromboembolism (PTE), spiral computed tomography angiography of pulmonary vessels was done, revealing PTE. After initiation of anti-coagulants, her clinical condition and respiratory status improved significantly. We present a rare case of SLE where only lupus anti-coagulant test was abnormal while other tests, such as anti-cardiolipin antibody and anti-phospholipid antibody were normal. Therefore, we can conclude that clinical suspicion had the main role in diagnosis in our case, as it has in medicine.
\end{abstract}

Keywords: pulmonary thromboembolism, ACLA, systemic lupus erythematosus (SLE)

\section{Introduction}

Systemic lupus erythematosus (SLE) is an autoimmune disease, with multisystem involvement and a broad spectrum of clinical presentations involving many tissues (1). Pulmonary involvement is a common manifestation in SLE, and it occurs in 25 to $75 \%$ of cases (1). The clinical spectrum includes pneumonitis, hemorrhage, pulmonary hypertension, pleural effusion, and pneumothorax. The uncommon pulmonary presentations are involvement of the diaphragm (including shrinking lung syndrome), vasculitis, and pulmonary thromboembolism (1). Pulmonary thromboembolism (PTE) rarely has been reported as the manifestation of SLE. This may be because it is masked by other more common and familiar lesions of the lungs (2). Anti-phospholipid antibody syndrome (APS) is a rare disease, particularly in children (3). But by comparing the primary and secondary forms, we see that the secondary form of APS, which occurs in patients with autoimmune disorders, is more common (3). APS has different clinical manifestations, with one of them being pulmonary thromboembolism (3). Pulmonary embolism related to APS is a

\section{Corresponding author:}

Assistant Professor Dr. Amir Anushiravani, Department of Internal Medicine, Faculty of Medicine, Allergy Research Center, Shiraz University of Medical Sciences, Shiraz, Iran.

Tel: +98.9173160616, Fax:+98.7136474613, E-mail: amiranush23@yahoo.com

Received: August 16, 2015, Accepted: December 21, 2015, Published: February 2016

iThenticate screening: December 21, 2015, English editing: February 02, 2016, Quality control: February 06, 2016

(C) 2016 The Authors. This is an open access article under the terms of the Creative Commons Attribution-NonCommercialNoDerivs License, which permits use and distribution in any medium, provided the original work is properly cited, the use is non-commercial and no modifications or adaptations are made. 
rare manifestation, particularly in children (3). This paper presents and discusses a case of SLE in remission using medication, but even though developed pulmonary thromboemboli as the first manifestation of APS despite having a normal anti-cardiolipin antibody (ACLA).

\section{Case presentation}

\subsection{Clinical presentation}

A 13-year-old female was diagnosed with SLE two years ago based on four of the American College of Rheumatology classification criteria for SLE. She was being treated with a combination of hydroxychloroqine and prednisolone without a positive antibody predicting thromboembolism.

\subsection{History}

She presented with acute chest pain, shortness of breath, and a fever of $39{ }^{\circ} \mathrm{C}$ about two weeks prior to hospitalization. There was no history of trauma recalled by the patient or her family. She presented with pain, which was located in the right hemithorax and increased in intensity in supine position. She also had a dry cough and labored breathing, which was aggravated by inspiration.

\subsection{Physical exam}

On physical examination, the patient's body mass index (BMI) was 24.5 , her respiratory rate was $35 /$ min, heart rate was $90 / \mathrm{min}, \mathrm{BP} 120 / 90 \mathrm{mmHg}$, and $\mathrm{O}_{2}$ sat. $92 \%$ in room air. No chest deformity was detected. On chest percussion, the lower half of the right lung had dullness. On chest auscultation, breath sounds had decreased in the lower right lung, and she had tenderness in her costophrenic angle. The heart and abdomen were normal on examination. All distal pulses were detectable, and the patient had no clubbing.

\subsection{Laboratory and imaging findings}

A chest X-ray was obtained, which was in favor of a consolidation in the lower lobe of the right lung, as shown in Figure 1. Also, minimal bilateral pleural effusion was confirmed in chest sonography. Electrocardioghraphy was normal. Laboratory tests were as follows: $\mathrm{WBC}=17200(\mathrm{PMN} \mathrm{83 \%} \mathrm{lymphocyte} 15 \%)$, hemoglobin $=7.9, \mathrm{mcv}=$ 57 , platelet (Plt) count $=485000, \mathrm{ESR}=114 \mathrm{~mm} / \mathrm{h}, \mathrm{CRP}=150$, anti-dsDNA $=200 \mathrm{U} / \mathrm{ml}$ (positive $>24$ ), anticardiolipin antibody (ACLA) $=5.8 \mathrm{U} / \mathrm{ml}$ (positive $>24$ ), and lupus anti-coagulant (LA) was $>120$ " (normal: 24-43"), Anti B2 glycoprotein (B2GP) IgG $=0.93 \mathrm{GPL} / \mathrm{m}$ (positive $>20$ ), Anti phospholipid Ab (APLA) $\mathrm{IgG}=1.2 \mathrm{U} / \mathrm{ml}$ (normal: up to 10) and anti-phospholipid $\mathrm{Ab} \operatorname{IgM}=0.8 \mathrm{U} / \mathrm{ml}$ (normal: up to 10), $\mathrm{BUN}=8$, Creatinin $=0.9$, urine analysis showed proteinuria $3+$ and hematuria $2+$, so urine was collected for $24 \mathrm{hr}$, and the results were as follows: volume $1,400 \mathrm{ml}$, protein $=1554 \mathrm{mg}$, creatinine $=4746$, calcium 23.8, D-Dimer value: 1822 (positive $>500)($ Table $1)$.

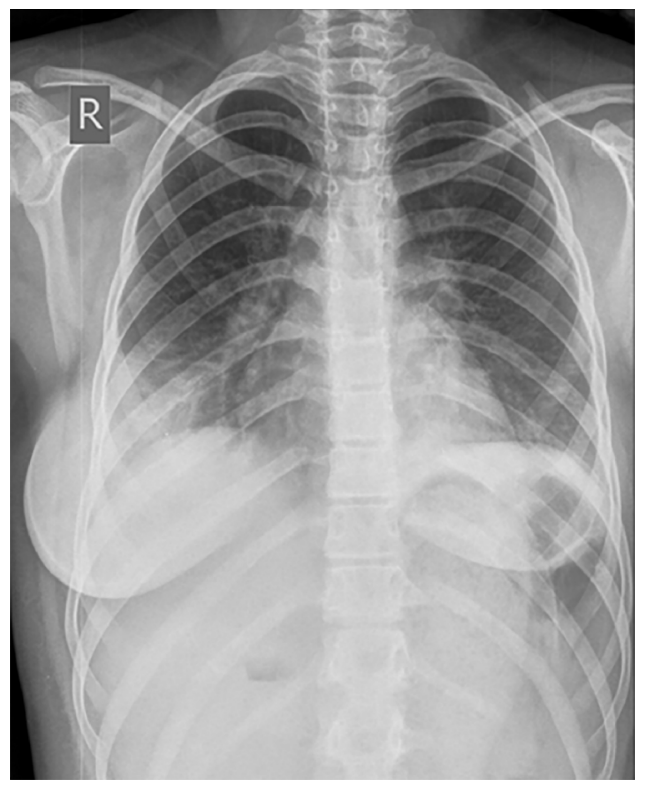

Figure 1. Chest $\mathrm{x}$-ray of the patient showing infiltration in right lung 
Table 1. Laboratory findings in our patient

\begin{tabular}{|c|c|c|c|}
\hline Test & Result & Test & Result \\
\hline WBC & $17,200 / \mu 1$ (neutrophils $83 \%$, lymph $15 \%$ ) & Anti-dsDNA & 200 (positive range $>24$ ) \\
\hline $\mathrm{Hb}$ & $7.9(\mathrm{~g} / \mathrm{dl})$ & ACLA & 5.8 (positive range $>24$ ) \\
\hline $\mathrm{MCV}$ & $57(\mathrm{fl})$ & LA & $>120$ ' (normal range: $24-43^{\prime \prime}$ ) \\
\hline Plt & 485000 & B2GP IgG & 0.93 (positive range $>20)$ \\
\hline ESR & $114(\mathrm{~mm} / \mathrm{hr})$ & APLA IgG & 1.2 (normal range up to 10 ) \\
\hline CRP & 150 & APLA IgM & 0.8 (normal range up to 10$)$ \\
\hline BUN & 8 & D-Dimer & 1822 (positive range $>500)$ \\
\hline $\mathrm{Cr}$ & 0.9 & Urinalysis & $3+$ proteinuria, $2+$ hematuria \\
\hline
\end{tabular}

dsDNA: double stranded DNA, ACLA: anti cardiolipin antibody, LA: lupus anti-coagulant, B2GP: Anti B2 glycoprotein, APLA: Anti phospholipid Ab

\subsection{Treatment and follow up}

An initial diagnosis of pneumonia was made and antibiotic therapy with clindamycin and ceftriaxone was started. No clinical improvement was seen three days later, and the patient presented with hemoptysis. So spiral high resolution computed tomography (HRCT) of the lung was obtained and patchy consolidation was noted bilaterally, especially in the lower lobes, as shown in Figure 2. With suspicion of pulmonary thromboembolism, Spiral CT Angiography of pulmonary vessels was done, revealing filling defects in the most distal part of the right pulmonary artery and descending branches of both pulmonary arteries suggestive of pulmonary thromboembolism (as shown in Figures 3 and 4). We started anticoagulation with enoxaparin and warfarin with a target INR between 2 and 3 , after labeling the patient with a diagnosis of anti-phospholipid syndrome probably secondary to systemic lupus erythematosus. An ultrasound color Doppler sonography of both lower extremities and an echocardiography were also done, and they were normal. We also treated her with intravenous cyclophosphamide and methylprednisolone.

\subsection{Outcome}

The patient became afebrile three days later, and, one week afterwards, her clinical condition and respiratory status improved significantly. The patient was seen one month later when she had no complaints and was in good health.

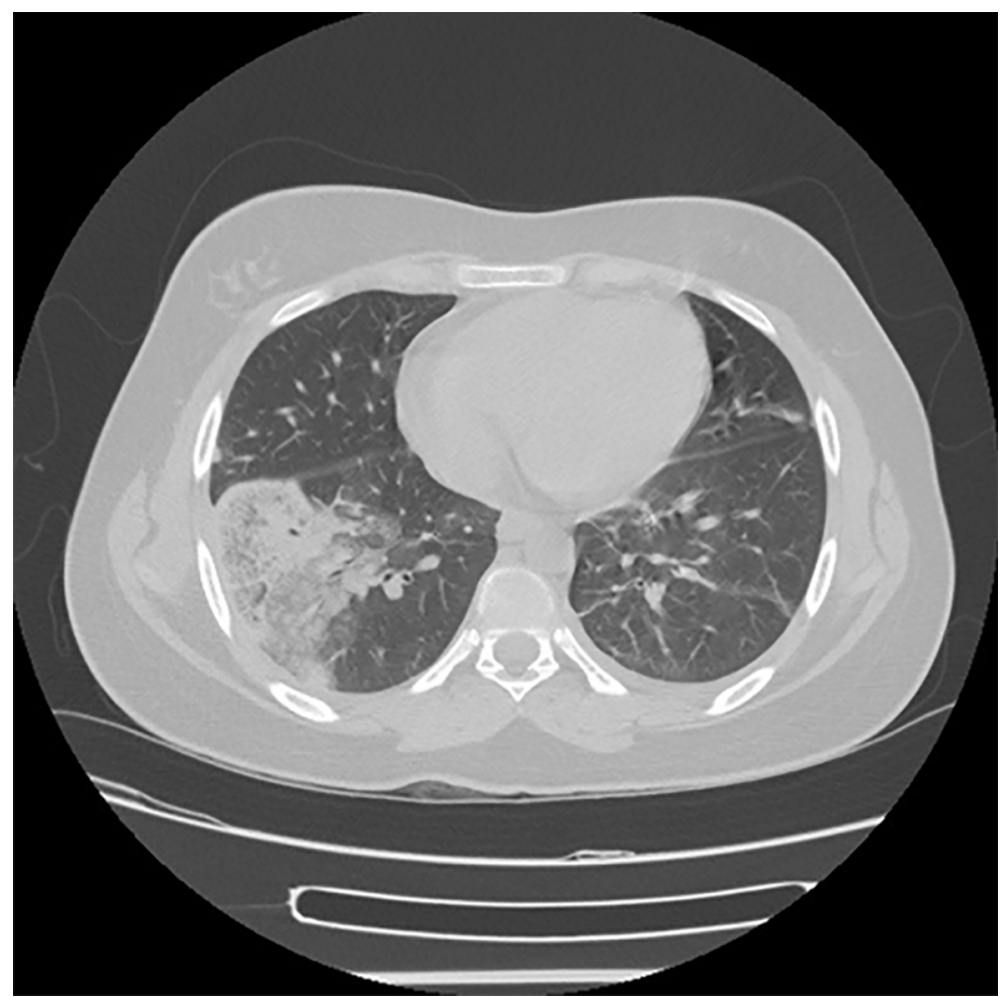

Figure 2. Chest CT scan of the patient showing patchy infiltration in right lower lobe 


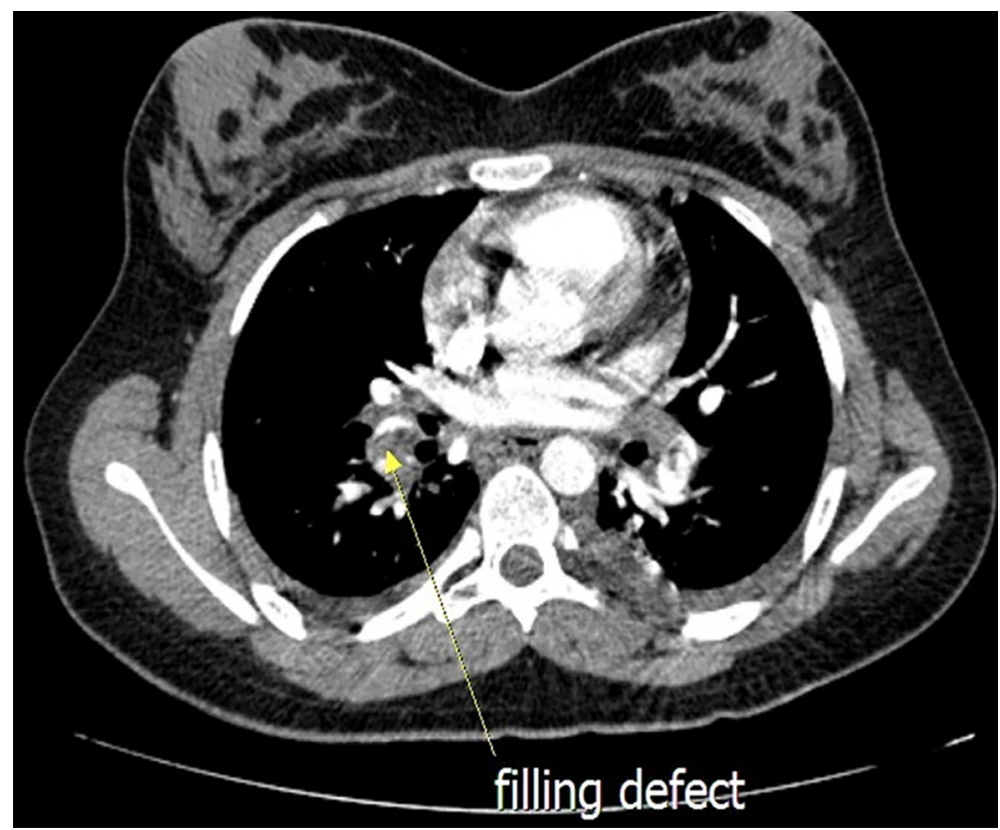

Figure 3. Spiral CT angiography revealing a filling defect in right main pulmonary artery

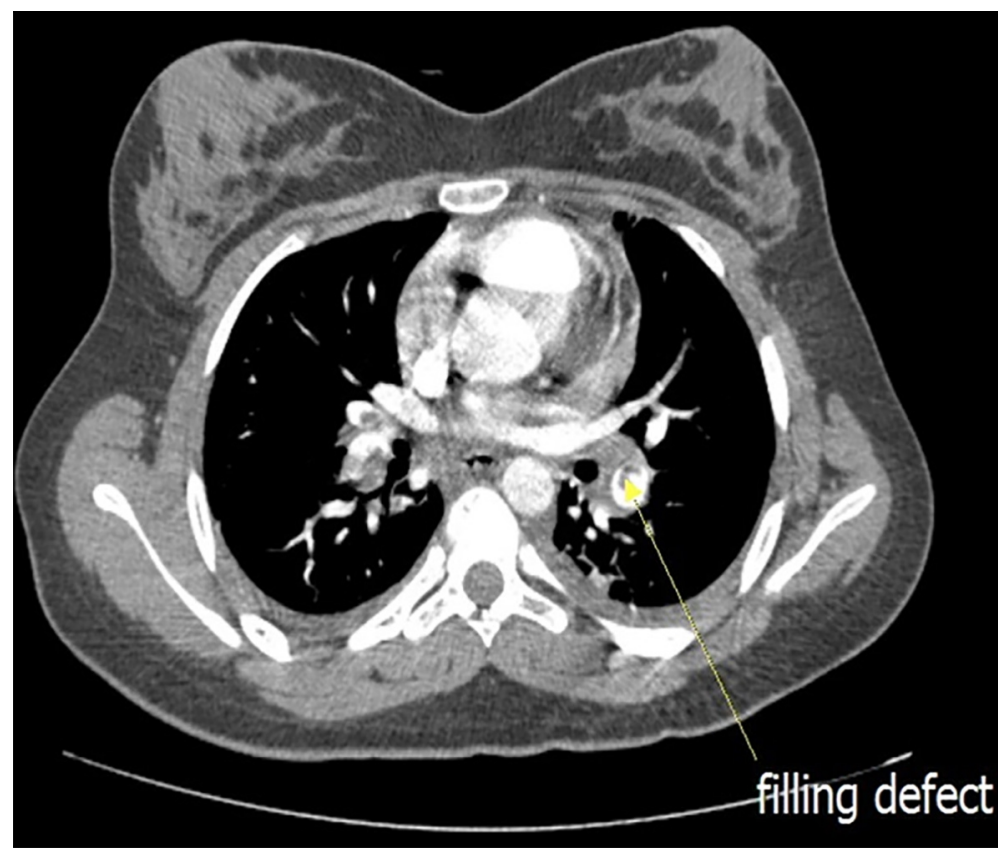

Figure 4. Spiral CT angiography revealing a filling defect in left main pulmonary artery

\section{Discussion}

SLE is an autoimmune inflammatory disease of unknown etiology that is characterized by damage to the tissues and cells due to deposition of pathologic immune complexes and auto antibodies. The clinical presentations of the illness are very different and determined by the presence of antibodies (4). Patients with SLE have an enhanced risk for thrombosis. Vessel thrombosis is a known morbidity in SLE, with a prevalence $>10 \%$. This prevalence could even exceed 50\% in susceptible patients with SLE (5). Among different causes of mortality in SLE patients, thromboembolism doesn't have an important role. The most common causes of death in this disease are renal involvement, infection, lupus encephalopathy, and pulmonary hypertension (10). Possible causes for the early and higher incidence of thrombosis could be the high levels of disease activity, a higher inflammatory state or circulating 
immune complexes, and cytotoxic antibodies (6). The most significant risk factor for thromboembolic events is the presence of circulatory anti-phospholipid anti-bodies in patients with SLE. Environmental factors seem to contribute to induction of such an autoantibody. Additional factors, such as infections, hormones, vitamins and sun exposure, are important in the production of APS and SLE (11). Antiphospholipid antibodies are a heterogeneous group of antibodies, in which a majority react with proteins bound to phospholipids $(4,7)$ and their presence can be determined by assessing the anticardiolipin antibodies (ACLA), lupus anticoagulant (LA), and beta2-glycoprotein I antibody in the patient's serum $(5,7)$. A high percentage of patients with SLE have circulating antiphospholipid antibodies (4). According to Shah et al.'s study, 61\% of the patients with SLE had anti phospholipid antibodies, and $51 \%$ of patients with positive APLA had a definitive anti-phospholipid syndrome. These results indicate that half of the SLE patients can develop complications related to thrombosis at any time throughout the course of their diseases $(7,8)$. Long, A. A. et al.'s study showed a statistically significant association between persistently high ACLA assays and thromboembolic events (9). Another study in 2007 by Tarr, showed that lupus anticoagulant had the strongest correlation with APS and was a better predictor of clinical thrombotic manifestations (7). We have described a patient who had pulmonary thromboembolism despite not having a circulatory ACLA (a common indicator of APLA) and while her disease was in remission using hydroxychloroquine and a low dose of prednisolone. Opposed to the results of previous studies, hydroxychloroquine has been demonstrated to reduce the disease-related complications and mortality, and it may also reduce the thrombotic risk (5). Patients with auto immune diseases are usually prone to thrombotic events in older ages (4), whereas, in our case, pulmonary embolism was seen in an adolescent. Pulmonary embolism due to SLE usually occurs in the active phase of the disease (2), similar to our case, because her anti-dsDNA and ESR were high, and we didn't find any source of infection.

\section{Conclusions}

SLE patients are prone to thrombosis formation, which is multifactorial. A classified approach to thrombosis risk factors is very important in the management of patients with SLE. Common risk factors of thromboembolism should be assessed in every visit and treated strictly. APLA should be screened in each SLE patient with alloantibody related to thrombotic event and an active disease, especially lupus nephritis, must be treated immediately.

\section{Acknowledgments:}

This study was based on a rare case presenting with SLE and PTE. There are no funders to report for this submission. We would like to acknowledge the Immunology and Allergy Ward of Nemazee Hospital and Shiraz University of Medical Sciences for giving us access to our patient's records and letting us report them.

\section{Conflict of Interest:}

There is no conflict of interest to be declared.

Authors' contributions:

All authors contributed to this project and article equally. All authors read and approved the final manuscript.

\section{References}

1) Benseler SM, Silverman ED. Systemic lupus erythematosus. Pediatr Clin North Am. 2005; 52(2): 443 -67. PMID: 15820375.

2) Gladman DD, Urowitz MB. Venous syndromes and pulmonary embolism in systemic lupus erythematosus. Ann Rheum Dis. 1980; 39(4): 340-3. PMID: 7436559.

3) Müller C, Willaschek C, Borst M, Buchhorn R. Recurrent pulmonary embolism in a boy with antiphospholipid syndrome. Case Reports in Clinical Medicine, 2, 310-314. 2013; 2(5): 310-14. doi: 10.4236/crcm.2013.25083.

4) Avcin T, Cimaz R, Silverman ED, Cervera R, Gattorno M, Garay S, et al. Pediatric antiphospholipid syndrome: clinical and immunologic features of 121 patients in an international registry. Pediatrics. 2008; 122(5): e1100-7. doi: 10.1542/peds.2008-1209, PMID: 18955411.

5) Afeltra A, Vadacca M, Conti L, Galluzzo S, Mitterhofer AP, Ferri GM, et al. Thrombosis in systemic lupus erythematosus: congenital and acquired risk factors. Arthritis Rheum. 2005; 53(3): 452-9. doi: 10.1002/art.21172, PMID: 15934123.

6) Manger K, Manger B, Repp R, Geisselbrecht M, Geiger A, Pfahlberg A, et al. Definition of risk factors for death, end stage renal disease, and thromboembolic events in a monocentric cohort of 338 patients with 
systemic lupus erythematosus. Ann Rheum Dis. 2002; 61(12): 1065-70. doi: 10.1136/ard.61.12.1065. PMID: 12429536, PMCID: PMC1753955

7) Tarr T, Lakos G, Bhattoa HP, Shoenfeld Y, Szegedi G, Kiss E. Analysis of risk factors for the development of thrombotic complications in antiphospholipid antibody positive lupus patients. Lupus. 2007; 16(1): 3945. doi: 10.1177/0961203306074767, PMID: 17283584.

8) Shah NM, Khamashta MA, Atsumi T, Hughes GR. Outcome of patients with anticardiolipin antibodies: a 10 year follow-up of 52 patients. Lupus. 1998; 7(1): 3-6. PMID: 9493141.

9) Berube C, Mitchell L, Silverman E, David M, Saint Cyr C, Laxer R, et al. The relationship of antiphospholipid antibodies to thromboembolic events in pediatric patients with systemic lupus erythematosus: a cross-sectional study. Pediatr Res. 1998; 44(3): 351-6. doi: 10.1203/00006450199809000-00014, PMID: 9727712.

10) Fei Y, Shi X, Gan F, Li X, Zhang W, Li M, et al. Death causes and pathogens analysis of systemic lupus erythematosus during the past 26 years. Clin Rheumatol. 2014; 33(1): 57-63. doi: 10.1007/s10067-0132383-3.

11) Agmon-Levin N, Shoenfeld $Y$. The spectrum between antiphospholipid syndrome and systemic lupus erythematosus. Clin Rheumatol. 2014; 33: 293-5. doi: 10.1007/s10067-014-2486-5. 